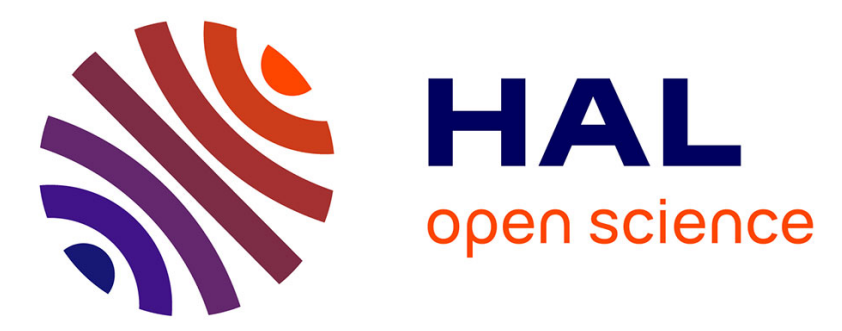

\title{
Near-field observation of surface plasmon polariton propagation on thin metal stripes
}

\author{
J. C. Weeber, J. R. Krenn, Alain Dereux, B. Lamprecht, Y. Lacroute, J. P. \\ Goudonnet
}

\section{- To cite this version:}

J. C. Weeber, J. R. Krenn, Alain Dereux, B. Lamprecht, Y. Lacroute, et al.. Near-field observation of surface plasmon polariton propagation on thin metal stripes. Physical Review B: Condensed Matter and Materials Physics (1998-2015), 2001, 64 (4), pp.045411. hal-00472593

\section{HAL Id: hal-00472593 https://hal.science/hal-00472593}

Submitted on 12 Apr 2010

HAL is a multi-disciplinary open access archive for the deposit and dissemination of scientific research documents, whether they are published or not. The documents may come from teaching and research institutions in France or abroad, or from public or private research centers.
L'archive ouverte pluridisciplinaire HAL, est destinée au dépôt et à la diffusion de documents scientifiques de niveau recherche, publiés ou non, émanant des établissements d'enseignement et de recherche français ou étrangers, des laboratoires publics ou privés. 


\title{
Near-field observation of surface plasmon polariton propagation on thin metal stripes.
}

\author{
J-C. Weeber, J. R. Krenn ${ }^{(\dagger)}$, A. Dereux, B. Lamprecht ${ }^{(\dagger)}$, Y. Lacroute and J. P. Goudonnet \\ Laboratoire de Physique de l'Université de Bourgogne, Optique Submicronique, BP 47870, \\ F-21078 Dijon, France \\ (†) Institut für Experimentalphysik, Universität Graz,Universitätsplatz 5, A-8010 Graz, Austria
}

(May 22, 2001)

\begin{abstract}
We use a Photon Scanning Tunneling Microscope to probe the field of surface plasmon polaritons modes excited on finite width thin metal films (metal stripes). We first investigate the coupling between surface plasmons launched by a focused beam on a homogeneous thin film and the modes sustained by metal stripes of different widths. We show that, if the width of the metal stripe is about a few microns, a strong coupling with the stripe modes can be achieved at visible frequencies. A sharp transverse confinement of the field associated to the surface plasmon modes propagating on the metal stripe is unambiguously observed on the constant height Photon Scanning Tunneling Microscope (PSTM) images. The back-reflection of theses modes at the end of the stripe leads to a surface--wave interference pattern from which the wavelength of the stripe surface plasmon modes is directly measured. We finally demonstrate that metal stripes could be used for optical addressing purposes at the micron scale since a stripe with a triangularly shaped termination performs the focusing of the stripe surface plasmon field.
\end{abstract}


PACS:

78.66.-w Optical properties of specific thin films, surfaces, and low- dimensional structures

71.36.+c Polaritons

07.79.Fc Near-field scanning optical microscopes

Typeset using REVTEX 


\section{INTRODUCTION}

Surface Plasmon Polaritons (SPP) are electromagnetic modes existing at the interface between a metal and a dielectric material [1]. SPP modes can propagate along the metal-air interface over a finite length depending on the ohmic losses of the metal. Theoretically, a typical mean-free path of a few tens of micrometers can be achieved at visible frequencies. The most common technique to excite a SPP at a metal-air interface consists in evaporating a thin metal film onto an optically dense substrate. Illuminating through the substrate at an angle of incidence slightly larger than the critical angle for total internal reflection allows the wave-vector of the incident light to match the one of the SPP. This technique known as Attenuated Total Reflection (ATR) leads to an illumination of the thin film similar to the usual illumination conditions of a sample observed with a Photon Scanning Tunneling Microscope (PSTM) [2]. Therefore, the PSTM turns out to be an efficient tool to investigate, in direct space, SPP excited in the ATR configuration. During the last decade, several works have demonstrated the capabilities of the PSTM to probe SPP in the near-field zone. For example, the first direct observation of SPP propagation along a silver-air interface can be found in Ref. [3]. More recently, elastic SPP scattering on individual surface defects has been reported in Refs [4-6]. However, in all the previously cited works, the SPP was excited on thin metal films of infinite widths. Up to now very little is known about the near-field behavior of a SPP launched on a finite width thin metal film (metal stripe).

In this work, we use a PSTM to perform what we believe to be the first near-field observations of SPP modes excited, at a visible frequency, on a metal stripe with a width in the range of a few micrometers. The excitation of SPP modes on a metal stripe is not only of fundamental interest but may have also practical applications. Indeed, on the basis of theoretical results and of far-field measurements, it has been shown very recently that thin metal stripes, embedded in a homogeneous dielectric and supporting long-range SPP modes, could be used as waveguides at optical telecommunication frequencies [7-9]. In this work, since we are concerned with metal stripes deposited on a glass substrate, the excitation of 
long-range SPP modes is not expected. Accordingly, a propagation length large enough to be useful for large scale integrated optics purposes seems rather unrealistic. However, the structures we consider provide an easy route to achieve the optical addressing by a surfacewave of objects deposited on a substrate such as metal nano-particles or even molecules. In the context of near-field optical microscopy, these features may be of experimental interest to investigate the near-field response of single objects.

The paper is organized as follows. In section II, we describe the experimental set-up we have used to obtain the constant height PSTM images. In section III, we investigate the behavior of a SPP excited by the focused gaussian beam on a thin film area with lateral dimensions much larger than the incident spot. In section IV, the coupling between the modes sustained by stripes of different widths and a SPP launched on the homogeneous thin film is considered. Specifically, the influence of an azimuthal or lateral misalignement of the incident beam with the axis of the stripe is discussed. Having in mind to use metal stripes for optical addressing applications, we consider in section $\mathrm{V}$ the capability of a triangulary shaped stripe termination to focus the field of a surface plasmon mode excited by shining the incident beam directly on a stripe. Finally, concluding remarks are given in section VI.

\section{EXPERIMENTAL BACKGROUND}

The PSTM setup we use in this work is described in Fig. 1. The sample is deposited on the hypotenuse side of a right angle BK 7 glass prism and illuminated by a monomode fiber focuser $(\mathrm{NA}=0.17$ and working distance $=20 \mathrm{~mm}$ ). The focuser is mounted on a rotation platform, allowing a fine tuning of the angle of incidence, and on a three axis micro-positioner which controls the location of the spot on the sample as well as the adjustment of the focus position. A $2.2 \mathrm{~mW}$ incident beam is obtained by injecting a $15 \mathrm{~mW}$ unpolarized red HeNe laser ( wavelength in vacuum: $\lambda=633 \mathrm{~nm}$ ) into the monomode fiber terminated by the focuser using a $\times 40$ microscope objective. A sharp tip, obtained by a standard heat-and-pull technique applied on a multimode optical fiber, is attached to a piezo-tube and approached 
to the immediate vicinity of the sample surface in order to collect the optical near-field resulting from the illumination of the sample. The optical signal picked-up by the fiber-tip is then converted by a photo-multiplier tube and amplified in a current/voltage amplifier. When the tip is scanned at constant height in the near-field zone, the signal collected by the probe provides the spatial distribution of the optical near-field intensity in a plane parallel to the sample surface.

All the experiments of this work have been performed with fiber-tips coated with $15 \mathrm{~nm}$ of chromium. Such a coating is necessary when dealing with highly scattering samples such as metallic structures in order to prevent a large contribution of radiative light to the optical signal collected by the tip. It has been recently reported that in the PSTM configuration, fiber-tips coated with a specific thickness of gold collect a signal that is proportional to the magnetic near-field intensity [10]. The present understanding of the detection process of these specific probes involves plasmon modes excitation at the very end of the tip. In our case, such a process is not expected since chromium does not exhibit surface plasmon resonance at visible frequencies. Indeed, the signal collected by the chromium coated tips is found to be of the same kind as the one detected by a purely dielectric tips, namely proportional to the electric near-field intensity [10-12]. The chromium layer allows also to use the fiber tip as a Scanning Tunneling Microscope (STM) probe if the surface of the sample is conductive.

The samples we consider are microfabricated on a conductive indium-tin-oxide (ITO) doped glass substrate such that the tunnel current can be used as the control signal during the tip-sample approach. Once a tunnel current is detected, an electronic circuit is activated in the feedback line. This circuit controls the tip-sample distance by changing the voltage applied on the $\mathrm{Z}$ electrode of the piezo-tube. If we assume that the sample surface position is given by the detection of the STM signal, the absolute distance between the tip and the sample is known and can be controlled with an accuracy better than $10 \mathrm{~nm}$ just by monitoring the $\mathrm{Z}$ electrode voltage. Using this procedure, we can achieve a fine approach of the tip and perform a constant height PSTM image very close to the sample. 


\section{SURFACE PLASMON POLARITON EXCITATION WITH A FOCUSED BEAM}

The first sample we consider has been designed to investigate the coupling between the surface plasmon modes supported by a metal stripe and a classical SPP excited on a homogeneous thin film area. The sample has been realized by standard electron-beam lithography technique on a ITO glass substrate covered with a spin-coated, $200 \mathrm{~nm}$ thick, polymethylmeta acrylate (PMMA) layer. After the lithography process, a silver film with a thickness of $60 \mathrm{~nm}$ has been deposited by sputtering onto the substrate. The root mean square (RMS) roughness of the silver film as determined by Atomic Force Microscope (AFM) imaging has been found to be about $2.5 \mathrm{~nm}$. After the lift-off of the PMMA layer, the sample shown in Fig. 2a has been obtained. The sample consists of a large thin film area to which several silver stripes of different widths are connected. The lower face of the substrate has been optically connected to the glass prism using an index matching oil such that the SPP at the silver-air interface can be excited in the ATR configuration. If the polarizer mounted on the focuser is rotated to align the electric field of the incident beam with the plane of incidence (TM polarization), the SPP excitation on the thin film area is effective at an angle of incidence of about $43^{\circ}$. Because we use a focused beam illumination, the SPP excitation manifest itself as a dark line in the circular reflected spot observed at the exit face of the prism. Obviously, this dark line is due to the absorption of the incident light travelling with a parallel component of the wave-vector that matches the one of the SPP.

The two images shown in Fig. 3a and Fig. 3b have been obtained for an incident beam respectively focused on the surface of the ITO substrate and on the homogeneous silver thin film area. In both cases, the beam propagates from the right towards the left side in a plane of incidence parallel to the $x$ axis and impinges on the surface of the sample with an angle of incidence of $43^{\circ}$. If located on the ITO substrate (Fig. 3a), the spot resulting from the total internal reflection of the incident beam exhibits an almost circular shape. This image, as well as all the PSTM images presented in this work, is normalized with respect to 
the maximum intensity detected during the scanning. In Fig. 3a, the slight elongation due to the oblique incidence illumination leads to a spot size with a FWHM of $10 \mu \mathrm{m}$ in the $x$ direction and $7 \mu \mathrm{m}$ in the $y$-direction. To obtain Fig. 3b, the spot has been moved on the silver film at a distance of about $20 \mu \mathrm{m}$ from the film edge (delimited by the white dashed line). The spot appears now cleary elliptical and asymmetric as shown by the cross-cut displayed in Fig. 3c. This behavior, first observed in the near-field by Dawson et al. [3], is attributed to the propagation of the SPP along the silver-air interface. In order to improve the visualization of the spot, the gray scale in Fig. 3b has been saturated to $20 \%$ of the full scale. It turns out that such a saturation is necessary because the angular width of the incident beam we use is much larger than the one of the SPP resonance. Indeed, this smears out the vizualisation in top view images of the SPP propagation since only a small amount of the incident light contributes to the SPP excitation.

Outside the launch site, the exponential decay of the near-field intensity allows to estimate the 1/e SPP propagation length $\left(\mathrm{L}_{S P P}\right)$ to $6.0 \mu \mathrm{m}$. This value is about two times smaller than the values previously measured on similar systems $[13,3]$. It is well known that the presence of a sulphide layer on the silver film, the roughness of this thin film or even the ohmic losses of the metal (which depends on the deposition process) can dramatically influence the value of $\mathrm{L}_{S P P}$. Since our goal in this work is not to optimize the SPP propagation, the value of $\mathrm{L}_{S P P}$ we have obtained is found to be convenient for our purposes and will serve as a reference to be compared with the propagation lengths measured over the stripes. Despite this rather low $\mathrm{L}_{S P P}$, one can see that the SPP reaches the film edge after a $15 \mu \mathrm{m}$-long path with an amplitude large enough to be detected. Indeed, an enhanced near-field signal is observed right above the film edge. Such an enhancement results from the inelastic SPP scattering at the step discontinuity between the silver film and the ITO substrate [14-16] and does not occur in the case of a smooth metal-dielectric transition [3]. 


\section{METAL STRIPES EXCITATION WITH A SURFACE PLASMON POLARITON}

In this section, we focus on the coupling between a SPP launched on the homogeneous thin film area and the stripe surface plasmon (SSP) modes sustained by a metal thin film of finite width.

The first silver stripe we consider has a width of $2.5 \mu \mathrm{m}$ and a length of $18 \mu \mathrm{m}$ (Fig. $2 \mathrm{~b}$ ). The image displayed in Fig 4a shows the optical near-field intensity distribution measured when the incident spot is located on the homogeneous thin film in front of the stripe at a distance of about $10 \mu \mathrm{m}$ from the film edge. In the right part of Fig $4 \mathrm{a}$, occupied by the homogeneous thin film, we recognize the elliptical spot characteristic of thin film SPP excitation. The scattering of the weak diffraction rings surrounding the incident spot, makes visible the thin film edge corresponding to the line $X=0.0 \mu \mathrm{m}$. A bright spot (marked by the white arrow) is observed at the termination of the stripe and can be intuitively attributed to the scattering of the surface plasmon propagating on the metal stripe. In order to check this assumption, the lateral position of the incident spot has been shifted by $15 \mu \mathrm{m}$ and a PSTM image (Fig. 4b) has been taken over the same sample area than the one of Fig 4a. In Fig. 4b, the bright spot at the termination of the metal stripe is not visible anymore so that we conclude that no SSP excitation occurs on the metal stripe with this last illumination condition. The same conclusion arises when an angular misalignement is introduced between the long axis of the metal stripe and the plane of incidence. The image shown in Fig. 5 has been obtained with the long axis of the stripe rotated at $45^{\circ}$ with respect to the propagation direction of the SPP. On this image, we just observe the scattering of the SPP at the thin film edge together with a weak surface-wave interference around thin film corner located in front of the incident spot. Finally, to give a last evidence of the SSP excitation, let us emphasis that, with a spot located as in Fig. 4a, no significant near-field signal was found above the stripe with an incident beam TE polarized.

All PSTM images we have discussed up to now have been taken over a large area of 
several hundreds $\mu \mathrm{m}^{2}$. For all these images, the typical tip-sample distance was set to be about $350 \mathrm{~nm}$ using the procedure previously described. This value has been choosen small enough to allow the detection of a near-field signal but at the same time large enough to prevent from a tip crash during the constant height scanning over a sample that could be slightly tilted with respect to the scanning plane. However, as the scanning area is reduced, the tip-sample distance can be safely decreased. We show in Fig.6 a series of PSTM images recorded over the $2.5 \mu \mathrm{m}$-wide stripe for decreasing scanning areas and tip-sample distances. Fig. 6a shows the behavior already discussed for Fig. 4a. By zooming on the stripe and by reducing the tip-sample distance to $100 \mathrm{~nm}$, we observe in Fig. 6b a neat standing wave pattern on both the homogeneous thin film area and the stripe. The interference pattern appears even more cleary in Fig. 6c at the end of the stripe if the tip-sample distance is reduced to less than $50 \mathrm{~nm}$. Such an interference pattern obviously shows the interaction of the SSP propagating on the metal stripe and the one back-reflected at its termination.

The curve displayed in Fig. 7a corresponds to a cross-cut of Fig. 6c taken perpendiculary to the long axis of the stripe. The most remarkable feature brought to the fore on this crosscut is the lateral confinement of the SSP mode to the width of the stripe. The two dashed lines in Fig. 7a represent the edges of the stripe. Note that the location of these two lines with respect to the SSP's profile is rather arbitrary since we didn't perform simultaneous topographic and near-field optical imaging. However, assuming that the maximum intensity is detected at the center of the stripe, one can see that the near-field signal over the edges drops to an almost null value. Qualitatively, this behavior is in agreement with the spatial distribution of the electric field associated with the modes sustained by a metal waveguide embedded in a homogeneous dielectric and exhibiting only one maximum along the direction perpendicular to the stripe axis (see for example the modes labelled $\mathrm{as} \mathrm{sa}_{b}^{1}$ or $\mathrm{ss}_{b}^{1}$ in Ref. [7]).

The length of the stripe we consider is less than eight times larger than its width, however, in a first approximation, we can assume that the field of the SSP propagating on our finitelength metal stripe varies along the propagation dimension as the modes existing on an infinitly long stripe [7]. With this assumption, the electric field components of the SSP 
propagating towards the positive values of $x$ can be written as:

$$
\mathcal{E}_{\delta}(x, y, z)=\mathcal{E}_{\delta}(y, z) \exp (-(\alpha+i \beta) x)
$$

where $\mathcal{E}_{\delta}$ denotes the amplitude of any electric field component $(\delta=\mathrm{x}, \mathrm{y}, \mathrm{z})$ of the SSP and $\alpha$ and $\beta$ are respectively the attenuation and the phase constant. Considering the interference of two counter-propagating SSP (representing the incident SSP and the back-reflected one), it is a simple matter to show that the intensity existing along the line $y=y_{0}$ located at a constant height $z_{0}$ over the stripe can be expressed as:

$$
\begin{array}{r}
I_{\delta}\left(x, y_{0}, z_{0}\right)=\mathcal{E}_{\delta}^{2}\left(y_{0}, z_{0}\right) \exp (-2 \alpha x)+r_{\delta}^{2} \exp \left(-4 \alpha x_{e}\right) \mathcal{E}_{\delta}^{2}\left(y_{0}, z_{0}\right) \exp (2 \alpha x) \\
+2 r_{\delta} \exp \left(-2 \alpha x_{e}\right) \mathcal{E}_{\delta}^{2}\left(y_{0}, z_{0}\right) \cos \left(2 \beta\left(x-x_{e}\right)-\phi_{\delta}\right)
\end{array}
$$

where $x_{e}$ denotes the position of the stripe termination and $r_{\delta}$ and $\phi_{\delta}$ are respectively the modulus of the reflection coefficient and the phase shift of the SSP electric field component $\delta$. The first and the second terms in Eq. 2 correspond respectively to an exponential damping and increase of the intensity upon the distance $x$ between the observation point and the launch site. If we suppose that the amplitude of the second term is negligible compared to the amplitude of the first one, the attenuation constant $\alpha$ is found to be related to the $1 / \mathrm{e}$ propagation length of the $\mathrm{SSP}\left(L_{\mathrm{SSP}}\right)$ by:

$$
\alpha=\frac{1}{2 \times L_{\mathrm{SSP}}}
$$

From the three PSTM images presented in Fig. 6, we estimate the attenuation constant of the SSP mode propagating on the $2.5 \mu \mathrm{m}$-wide stripe to $\alpha=0.077 \mu \mathrm{m}^{-1}\left(L_{\mathrm{SSP}}=6.5 \mu \mathrm{m}\right)$. Note that with such a value of $\alpha$, it is licit to neglect the second term in Eq. 2 since for the $18 \mu \mathrm{m}$-long stripe we use, even with a unitary reflection coefficient, the factor $r_{\delta}^{2} \exp \left(-4 \alpha x_{e}\right)$ leads to an amplitude of the second term more than 200 times smaller than the amplitude of the first one. Let us consider now the oscillating term in Eq. 2. From the argument of the cosine occuring in this term, one can see that the phase constant $\beta$ can be calculated via the relation: 


$$
\beta=\frac{\pi}{d_{\mathrm{SSP}}}
$$

if $d_{\mathrm{SSP}}$ denotes the periodicity of the interference pattern observed on the PSTM images. By averaging 20 periods on the cross-cut displays in Fig. $7 \mathrm{~b}$, we find a periodicity $d_{\mathrm{SSP}}=308$ $\mathrm{nm}$ corresponding to a phase constant $\beta=10.2 \mu \mathrm{m}^{-1}$. The most surprising feature of the image shown in Fig. $6 c$ is probably the visibilty of the interference pattern over a distance about tree times larger than the propagation length $L_{\mathrm{SSP}}$. However, this behavior is in agreement with Eq. 2 since the amplitude of the oscillating term does not depend on the propagation distance $x$. Therefore, as it can observed in Fig. 7b, the amplitude of the stationary wave pattern remains constant along the stripe. The large amplitude of the interferences compared to the monotonically attenuated intensity background suggests a quite large reflection coefficient at the end of the stripe in qualitative agreement with numerical computations of the SPP scattering by a 1D indentation in a homogeneous thin film $[17,18]$.

The previous experiment demonstrates that the propagation constant of a SSP can be deduced directly from the constant height PSTM images. In order to evaluate the dispersion of the complex propagation constant with respect to the width of the stripe, we analyse now the results obtained for a stripe with a width of $1.6 \mu \mathrm{m}$ ( length kept at $18 \mu \mathrm{m}$, see Fig. 2c). The PSTM images of this new sample are shown in Fig. 8. As in the previous case, the SSP excitation revealed by the detection of an optical near-field signal over the stripe can be observed in the large scanning image (Fig. 8a). However, the intensity of the scattering spot at the termination of the stripe is found to be much weaker than in the previous situation. Note that it is licit to compare the relative intensity in Fig. 6a and 8a since in both case, the normalization intensity is imposed by the incident spot. When reducing the scanning area and the tip-sample distance, we observe a spatial distribution of the near-field signal confined to the width of the stripe together with an intense spot at its termination (Figs. $8 \mathrm{~b}$ and $8 \mathrm{c}$ ). Since this spot is only clearly visible on the near-field images taken with a tipsample distance of a few tens of nanometers, we conclude that, the field at the end of the 
stripe is mostly evanescent. Therefore, unlike what has been observed on the $2.5 \mu \mathrm{m}$ wide stripe (see Figs. 6a and 6b), only a small amount of the field is scattered toward the vacuum when the SSP mode reaches the end of the stripe. A quite similar behavior has already been mentioned for surface defect supporting a localized surface plasmon resonance at a given frequency and exhibiting at the same frequency a small value of the vacuum scattering crosssection [19]. The fuzzy, low contrast, surface-wave interference pattern visible in Fig. 8b and $8 \mathrm{c}$ is unfortunatly to weak to allow an accurate measurement of the pattern periodicity. However, from these images, we find a propagation length $L_{S S P}=4.8 \mu \mathrm{m}$ significantly smaller than in the previous case $\left(\alpha=0.1 \mu \mathrm{m}^{-1}\right)$. The same experiments has been performed on a stripe with a width of $1 \mu \mathrm{m}$. The PSTM images obtained on this sample (Figs. 9a and 9b) comfirm that the excitation of a SSP is less efficient to propagate light as the width of the stripe decreases. As shown in Fig. 9b, the intensity on the stripe is strongly damped with the distance to the thin film edge. In this case, the attenation constant is about six time larger $\left(L_{S S P}=1.2 \mu \mathrm{m}, \alpha=0.42 \mu \mathrm{m}^{-1}\right)$ than for the $2.5 \mu \mathrm{m}$ wide stripe. Obviously, with such an attenuation, the amplitude of the SSP field at the termination of the stripe is so weak that no scattering spot or back-reflection can be observed in the PSTM images.

With the real part of dielectric function of silver taken equal to $\epsilon_{A g}^{\prime}=-15.86$ [20], the wavelength of the SPP at an infinite silver-air interface is found to be $\lambda_{S P P}=613 \mathrm{~nm}$ for an incident wavelength of $633 \mathrm{~nm}$ in vacuum. Therefore, the results obtained on the 2.5 $\mu \mathrm{m}$ wide stripe suggest that if the width of the stripe is about four times $\lambda_{S P P}$ the mode excited by the SPP and propagating on the stripe exibits characterictics close to those of the interface SPP itself. Indeed, the propagation length on this stripe is of the same order than the one estimated on the homogeneous thin film and furthermore the wavelength of the SSP mode $\left(\lambda_{S S P}=616 \mathrm{~nm}\right)$ is found to be almost equal to the wavelength of the interface surface plasmon $\left(\lambda_{S P P}=613 \mathrm{~nm}\right)$. If the width of the stripe is divided by two, the damping of the SSP mode becomes so large that the propagation of light at a scale of a few microns is not achievable anymore. On the basis of these results, we conclude that the excitation conditions of a "wide" (within the range given above) stripe are very close to the ones of a 
thin film SPP.

\section{FOCUSING STRIPE SURFACE PLASMON MODES FIELD}

The short propagation length of the SSP modes at visible frequencies prohibits the use of the thin metal stripe for macroscopic integrated optic purposes. However, the thin metal stripe provides an interface between a macroscopic source of illumination (the focused beam in our case) and objects deposited on a transparent substrate or even directly on the stripe. Accordingly, one can anticipate potential applications of the metal stripes to achieve the optical addressing at micron scale by a surface-wave of nanoscopic objects such as metal nanoparticles or molecules. With this goal in mind, the main challenge is the control of the SSP mode confinement. The previous results prove that the utilization of narrow stripes to achieve a good SSP confinement is not convenient since it leads at the same time to a drastic reduction of the propagation length. Recently, it has been reported in several works that 2Dwaves optical components relying on elastic multiple SPP scattering could be generated from an arrangement of large thin film indentations [4,5]. Obviously, a surface-wave lens would be of great help to perform the focusing of the SSP mode. Unfortunatly, a lens generated from indentations in the stripe seems rather inconceivable in our case since it would prevent SSP propagation. To overcome these difficulties, we have adopted the most basic solution that consists in using a "wide" stripe to keep the propagation length at a reasonable level and to structure the shape of the stripe termination in order to squeeze the lateral extension of the SSP mode. To this aim, a $3.5 \mu \mathrm{m}$ wide silver stripe with a triangularly shaped termination has been micro-fabricated. Since we known from the previous results that with such a width the SSP launching is effective for illuminations parameters close to those that correspond to the excitation of an infinite thin film SPP, the stripe has been excited with the focused beam shining directly on it at an angle of incidence of $43^{\circ}$. The constant height PSTM image shown in Fig. 10a has been obtained for an incident spot located about $30 \mu \mathrm{m}$ from the stripe end. The SSP propagation (from the top to the bottom) is clearly visible and leads to an intense 
scattering spot at the stripe termination. From the cross-cut displayed in Fig. 10b, we find a propagation length $L_{S S P}=7.0 \mu \mathrm{m}$ close to the one previously measured for the $2.5 \mu \mathrm{m}$ wide stripe. To probe the behavior of the SSP when encountering the stripe end (Fig. 11a), a PSTM image has been taken over a reduced scanning area (Fig. 11b). Far from the stripe end, we notice a standing wave pattern of the same kind than the one previously observed in Fig. 6c. By direct comparison with the AFM image of the stripe, the confinement of the SSP mode appears unambiguously. As shown in Fig. 11a, the stripe end is represented by a triangle with an opening angle of about $90^{\circ}$. On the right side of the stripe, a narrow silver wire resulting from a defect of the lithographic process can be observed. It turns out that this wire does not pertub significantly the SSP field. Within the triangular area, the PSTM image exhibits a two-dimensionnal interference pattern attributed to the interaction of the incident SSP with the two SSP reflected by each side of the triangle. At the very end of the stripe, we observe an intense localized spot due to foward scattering of the SSP presenting a lateral extension of less than one third of the stripe width $(\mathrm{FWHM}=1.0 \mu \mathrm{m}$ in the $x$ direction). Since in the case of a rectangular stripe termination the scattering spot extends over the entire width of the stripe (see Figs. 6c and 8c), we conclude that, by using a triangular termination, we have achieved the focusing of the SSP field.

\section{CONCLUSION}

In summary, we have used a PSTM set-up to demonstrate the feasibility of surface plasmon guiding by laterally confined thin films deposited on a glass substrate. To excite the SSP modes sustained by the stripes, we first use a SPP launched by a focused beam on a homogeneous thin film area whose lateral extension is much larger than the incident spot. If the width of the stripe is about four times the SPP wavelength, the propagation constant of the SSP mode is found to be close to the one of the SPP. Therefore, for stripe widths down to $2.5 \mu \mathrm{m}$ stripes, a good coupling between thin film SPP and the stripe modes can be achieved at visible frequencies. Once launched, the SSP mode is damped with propagation 
distance but, laterally, remains well confined all along the stripe. When reaching the stripe end, a quite large back-reflection of the SSP field leads to the creation of a stationary wave pattern exhibiting a constant amplitude all along the stripe. Finally, a direct excitation of the SSP mode by the incident focused beam has been demonstrated for a $3.5 \mu \mathrm{m}$ wide stripe. Using a triangularly shaped stripe termination, we have shown that a focusing of the SSP field could be achieved. On the basis of these results, many other situations as the splitting or the deviation of SSP can be considered. Experiments dealing with these topics are in progress and will be reported elsewhere. 


\section{REFERENCES}

[1] H. Raether, Surface Plasmons, Springer Tracts in Modern Physics, Vol 111 (Springer, Berlin, 1988).

[2] R.C. Reddick, R.J. Warmack, T.L. Ferrell, Phys. Rev. B, 39, 767 (1989).

[3] P. Dawson, F. de Fornel, J.P. Goudonnet, Phys. Rev. Lett., 72, 2927 (1994).

[4] S. I. Bozhevolnyi, F. A. Pudonin, Phys. Rev. Lett., 78, 2823 (1997).

[5] S. I. Bozhevolnyi, V. Coello, Phys. Rev. B, 58, 10899 (1998).

[6] I. I. Smolyaninov, D. L. Mazzoni, C. C. Davis, Phys. Rev. Lett., 77, 3877 (1996).

[7] P. Berini, Phys. Rev. B, 61, 10484 (2000).

[8] R. Charbonneau, P. Berini, E. Berolo, E. Lisicka-Shrzek, Optics Lett. 25, 844 (2000).

[9] P. Berini, Optics Express, 7, 329 (2000).

[10] E. Devaux, A. Dereux, E. Bourillot, J-C Weeber, Y. Lacroute, J-P Goudonnet, Ch. Girard, Phys. Rev. B, 62, 10504 (2000).

[11] J. R. Krenn, A. Dereux, J-C Weeber, E. Bourillot, Y. Lacroute, J-P Goudonnet, G. Schider, W. Gotschy, A. Leitner, F. R. Ausseneg, Ch. Girard, Phys. Rev. Lett., 82, 2590 (1999).

[12] J-C Weeber, E. Bourillot, A. Dereux, J-P Goudonnet, Y. Chen, Ch. Girard, Phys. Rev. Lett., 77, 5332 (1996).

[13] M. van Exter, A. Lagendijk, Phys. Rev. Lett., 60, 49 (1988).

[14] R. F. Wallis, A. A. Maradudin, G. I. Stegeman, Appl. Phys. Lett. 42, 764 (1983).

[15] H. A. Jamid, S. J. Al-Bader, IEEE Photonic Technology Letter, 7, 321 (1995).

[16] H. A. Jamid, S. J. Al-Bader, IEEE Photonic Technology Letter, 9, 220 (1997). 
[17] J. A. Sànchez-Gil, Appl. Phys. Lett., 73, 3509 (1998).

[18] U. Schröeter, S. Seider, S. Tode, D. Heitmann, Ultramicroscopy, 68, 223 (1997).

[19] A. V. Shchegrov, I. V. Novikov, A. A. Maradudin, Phys. Rev. Lett., 78, 4269 (1997).

[20] Handbook of Optical Constants of Solids, edited by D. Palik (Academic, New York, 1985). 


\section{FIGURES}

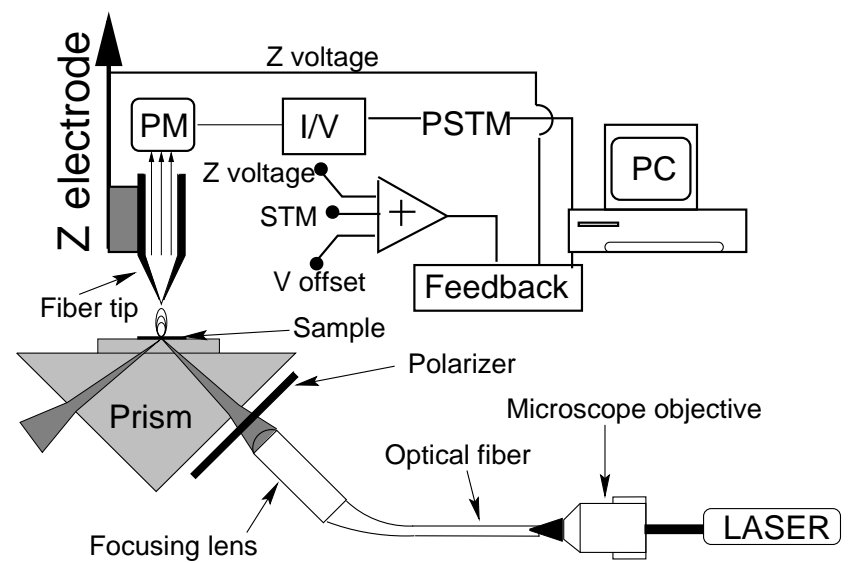

FIG. 1. Schematic view of the experimental PSTM setup. (PM): Photomultiplier, (I/V): Current/voltage amplifier, (details see text).
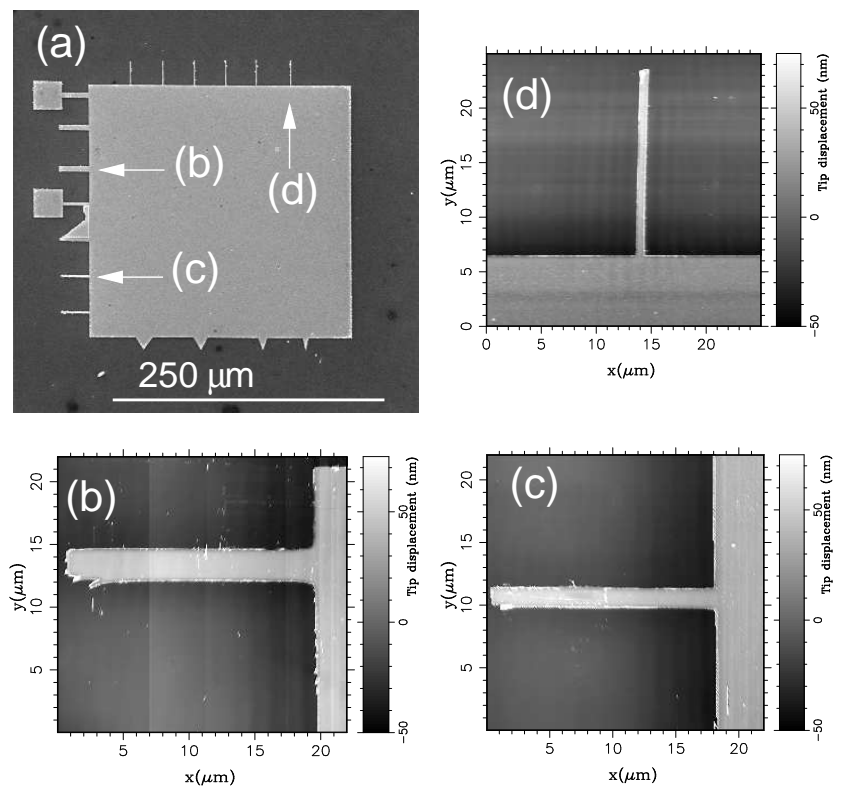

FIG. 2. (a) SEM image of the silver sample designed to study the coupling between interface SPP and the modes sustained by finite width thin metal films (stripes). Several stripes of different widths are connected to a large homogeneous thin film area of $250 \times 250 \mu \mathrm{m}^{2}$. Atomic force microscope images of the three stripes considered. (b) width $=2.5 \mu \mathrm{m}$. (c) width $=1.6 \mu \mathrm{m}$. (d) width $=1.0$ $\mu \mathrm{m}$ 


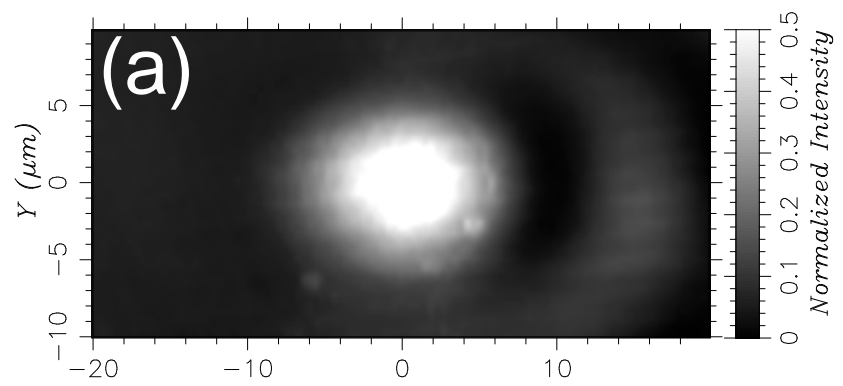

$X(\mu m)$
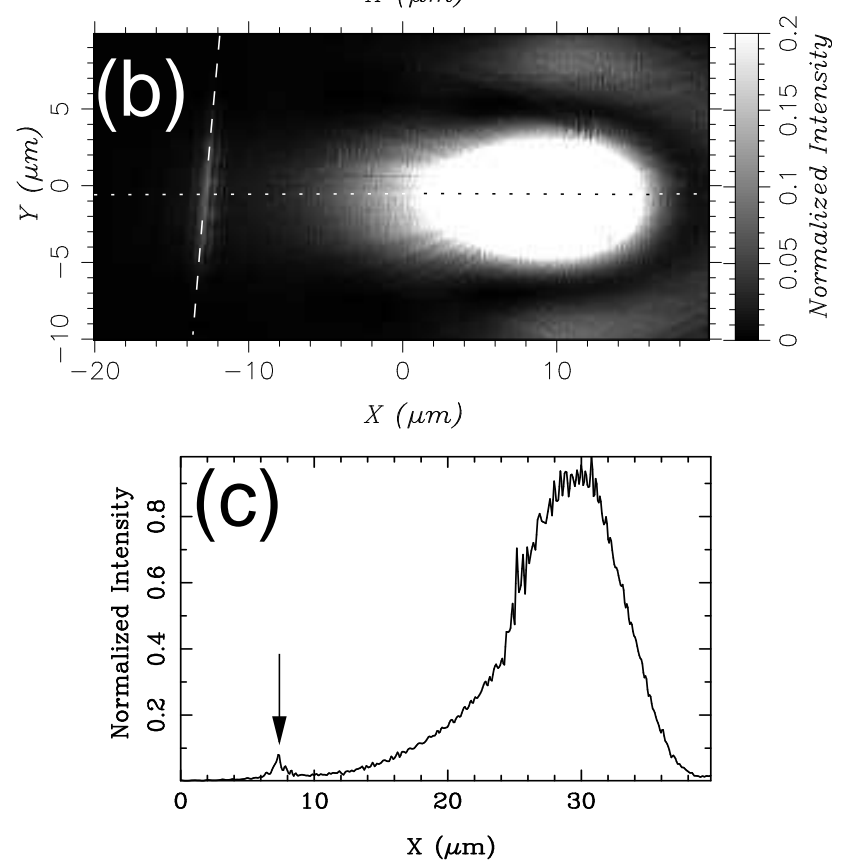

FIG. 3. PSTM image of the incident spot for an angle of incidence of $43^{\circ}$. (a) The beam is focused on the surface of the ITO doped glass substrate. (b) The spot has been moved on the homogeneous silver thin film area. The dashed line marks the thin film edge. (c) Cross-cut of (b) along the dotted line. The assymetric shape of the spot is due to the propagation of the SPP along the silver-air interface. Inelastic SPP scattering leads to an enhanced optical signal over the film edge (black arrow). 

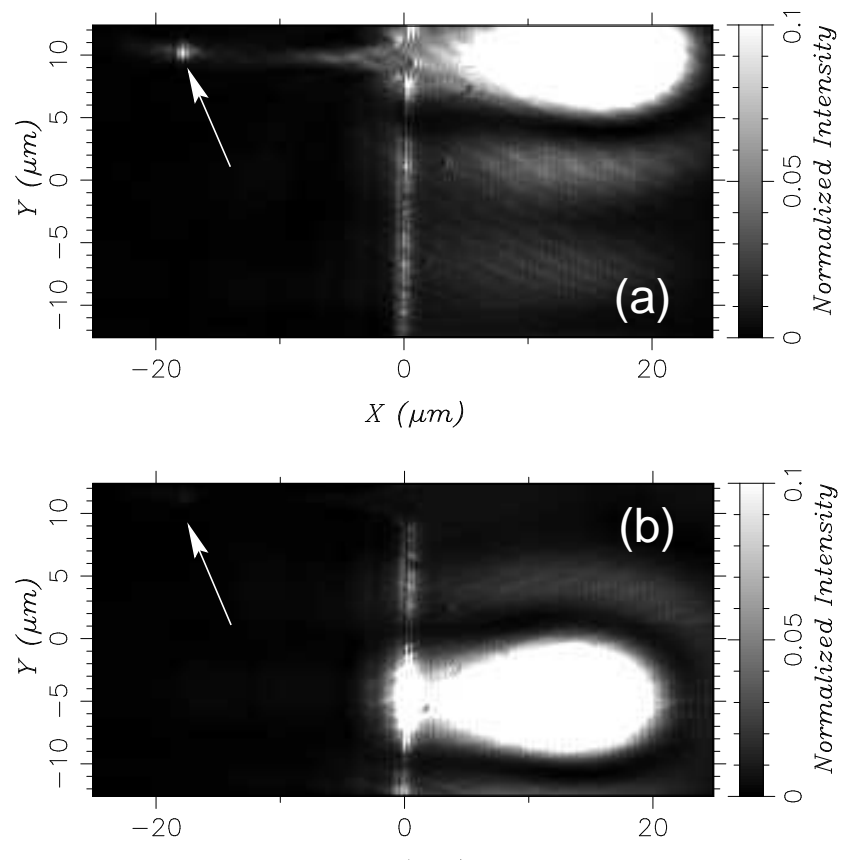

FIG. 4. PSTM image of the $2.5 \mu \mathrm{m}$ wide stripe excited by a SPP launched on the homogeneous thin film area. (a) The incident spot is located in front of the stripe. At the end of the stripe a scattering spot (white arrow) is visible. (b) The incident spot has been moved $15 \mu \mathrm{m}$ in the $y$ direction. The scanning area is the same than that of (a).

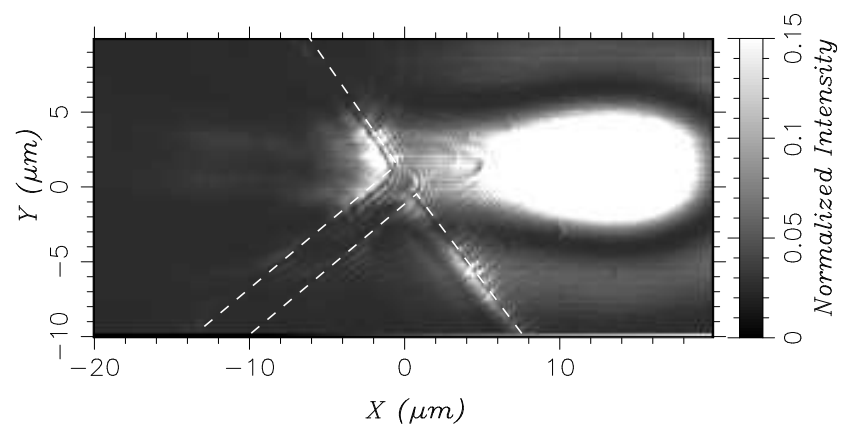

FIG. 5. PSTM image of the $2.5 \mu \mathrm{m}$ wide stripe when the plane of incidence is rotated $45^{\circ}$ with respect to the long axis of the stripe (the surface projection of the film edges and the stripe correspond to the white dashed lines). 

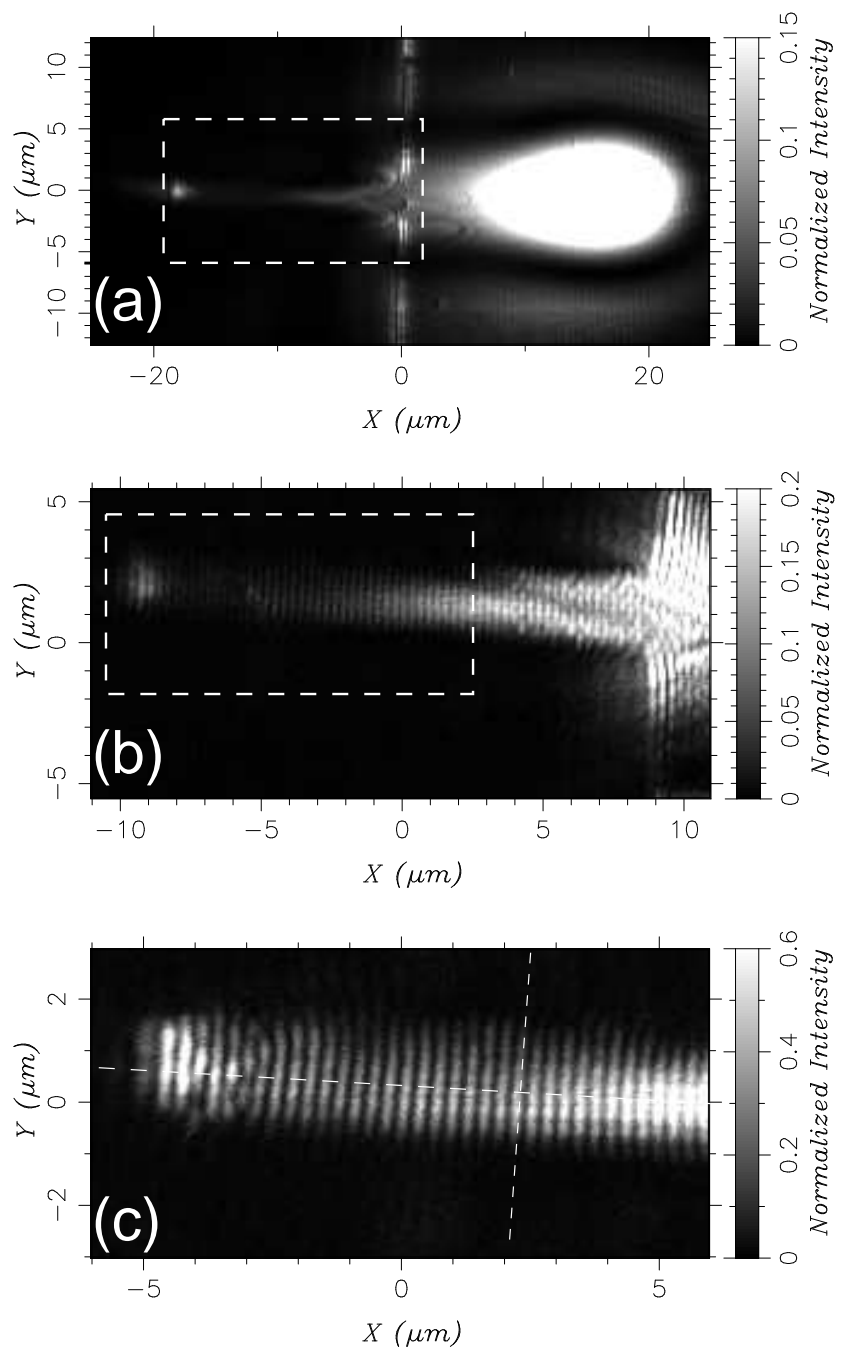

FIG. 6. PSTM image of the $2.5 \mu \mathrm{m}$ wide stripe (see Fig. 2b) excited as in Fig. 4a. (a) The tip-sample distance is about $350 \mathrm{~nm}$. (b) The tip-sample distance has been reduced to $100 \mathrm{~nm}$. (c) The tip-sample distance is less than $50 \mathrm{~nm}$. The size of images (b) and (c) corresponds to the area indicated by the box shown respectively in (a) and (b). 

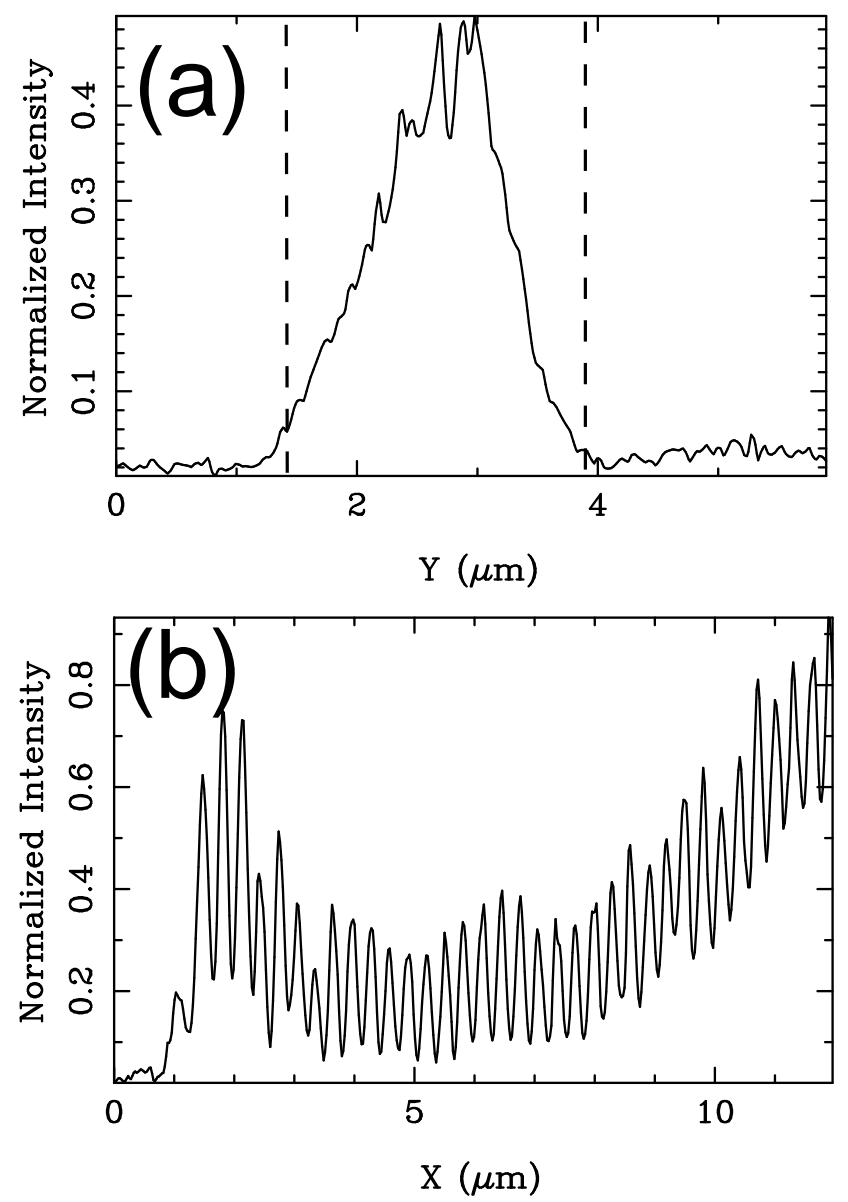

FIG. 7. Cross-cuts of Fig. 6c. (a) Along the width of the stripe. (b) Along the long axis of the stripe. The two cross-cuts lines are shown on Fig. 6c. 

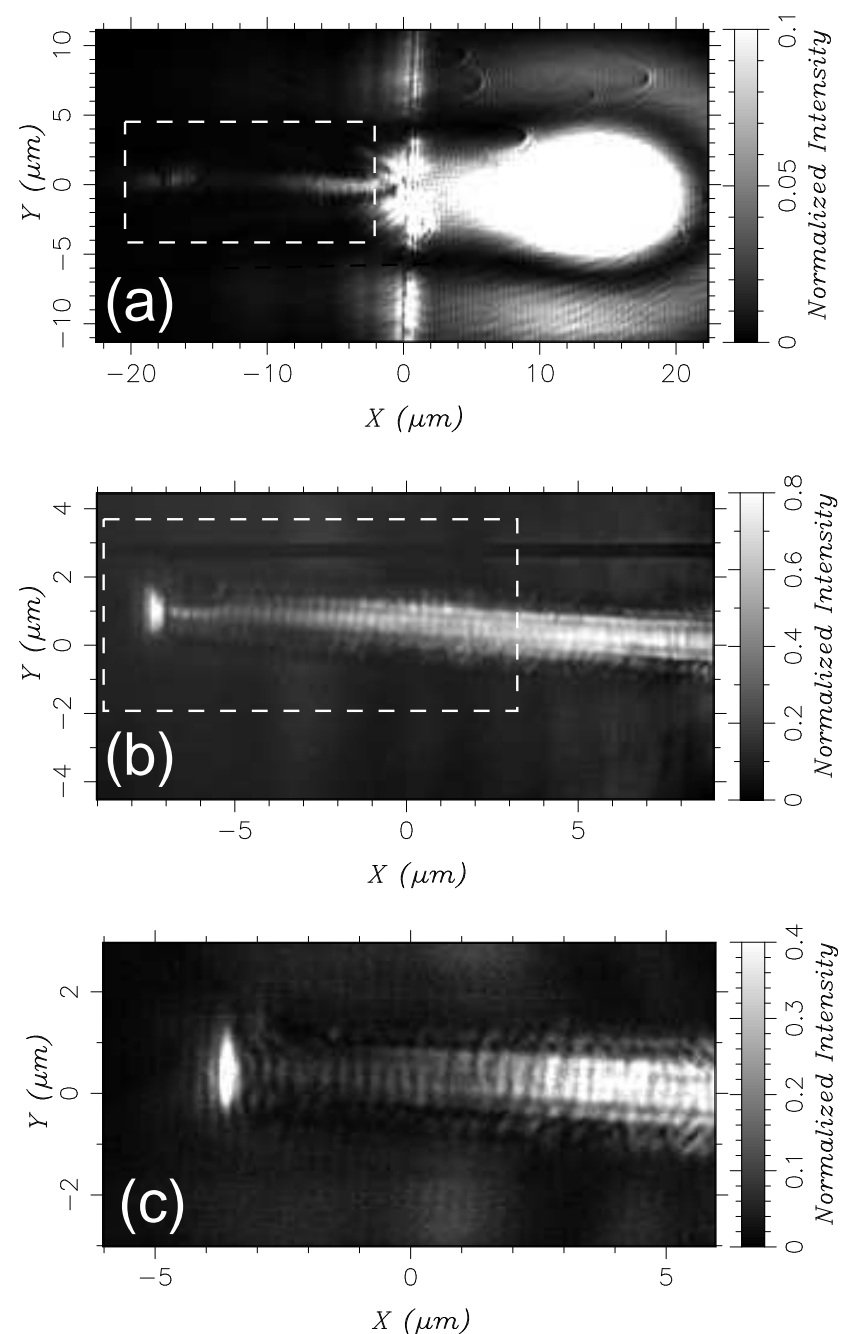

FIG. 8. PSTM images of the $1.6 \mu \mathrm{m}$ wide stripe (shown in Fig. 2c) excited by an interface SPP . The tip-sample distances for images (a) (b) and (c) are similar to thoses for corresponding images in Fig. 6. 

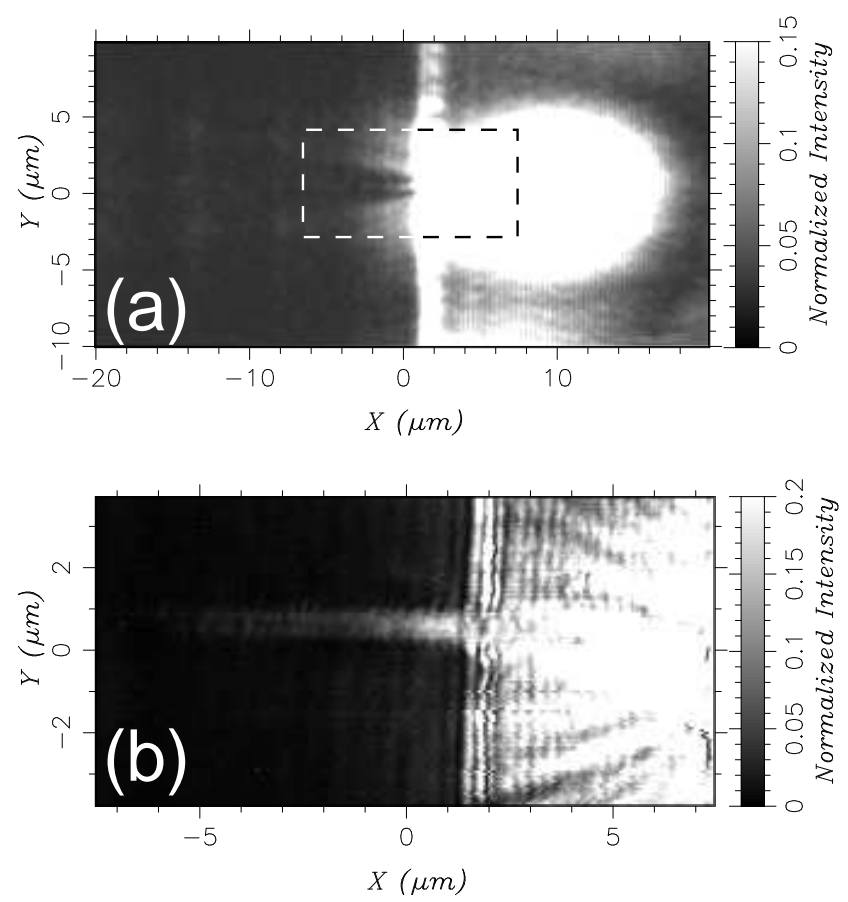

FIG. 9. PSTM images of the $1.0 \mu \mathrm{m}$ wide stripe (Fig. 2d) excited by an interface SPP. (a) Tip-sample distance around $350 \mathrm{~nm}$. (b) Zoom taken over the area shown in Fig. 9a with a tip-sample distance smaller than $50 \mathrm{~nm}$. 

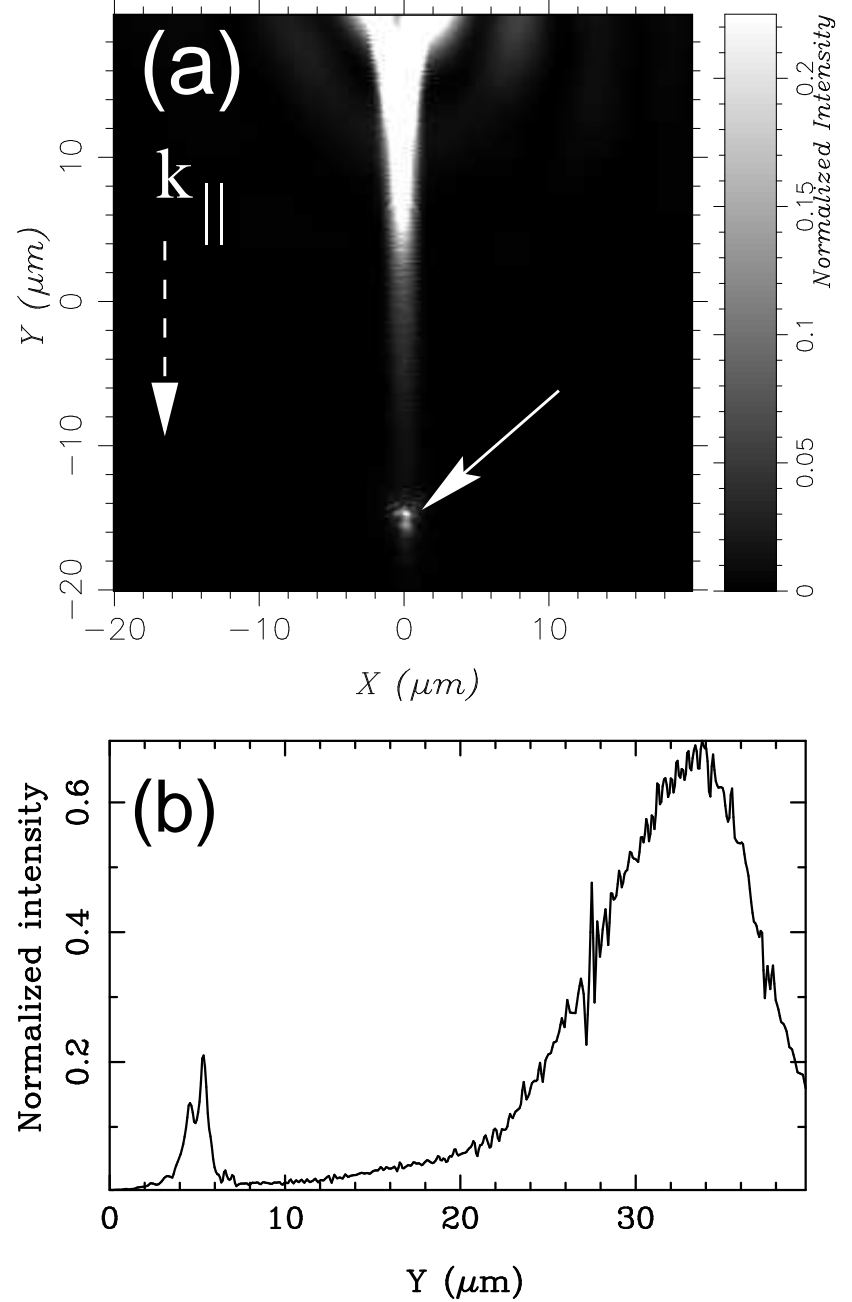

FIG. 10. (a) PSTM image of the $3.5 \mu \mathrm{m}$ wide stripe. The stripe end has been micro-structured to be triangular. The SSP mode excited on the stripe by direct illumination with the focused beam propagates from the top to the bottom of the image. The stripe end is shown by the white arrow. (b) Cross-cut of (a) along the $y$ direction $(\mathrm{x}=0.0 \mu \mathrm{m})$. 

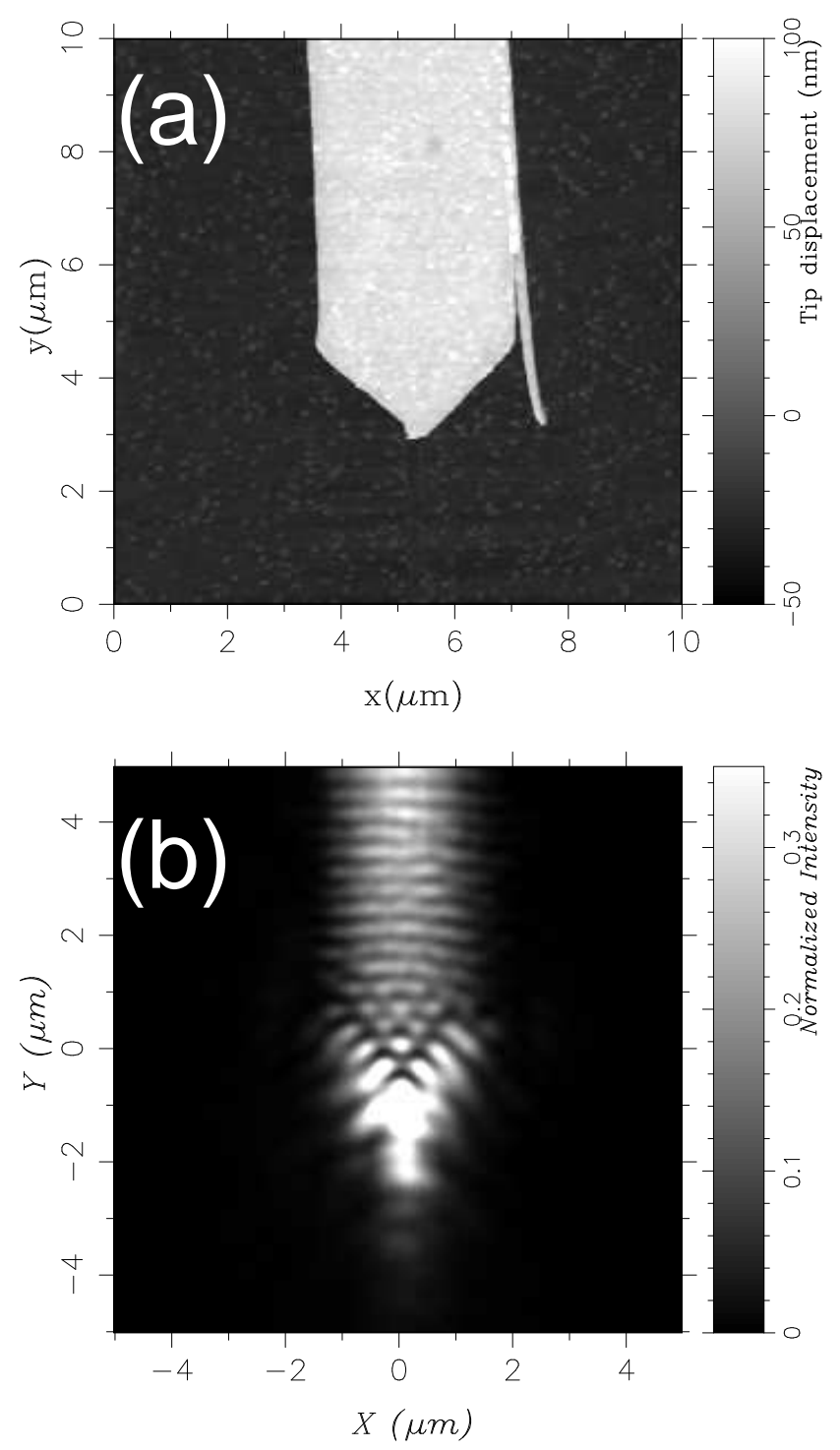

FIG. 11. (a) AFM image of the triangular termination of the $3.5 \mu \mathrm{m}$ wide stripe. (b) PSTM image taken over the triangular termination with the stripe excited as in Fig. 10a. 